\title{
PREAMPLIFYING CANTILEVERS FOR CONTACT RESONANCE MODE IMAGING
}

\author{
B. Zeyen ${ }^{1}$, B. Pittenger ${ }^{2}$, K. Virwani $^{2}$, K.L. Turner ${ }^{1}$ \\ ${ }^{1}$ University of California, Santa Barbara, California, USA \\ ${ }^{2}$ Veeco Metrology Inc., Santa Barbara, California, USA
}

\begin{abstract}
A novel preamplifying cantilever (PCL) design for scanning probe microscopes (SPM) that is capable of mechanically amplifying specimen movements is presented. The sample motions in both out-of-plane and in-plane directions are amplified by the PCL. The spatial resolution of the cantilever is in the nanometer scale and is comparable with existing SPM cantilevers. Some of the proposed applications are in the areas of ferroelectric domain imaging [1]-[5].

The amplification was demonstrated with measurements on ferroelectric materials- which included periodically poled lithium niobate (PPLN). Feasibility tests show that a 100x mechanical preamplification is possible with the current cantilever design, providing a much better signal to noise ratio on both amplitude and phase response measurements than the previous art (e.g. [8]).
\end{abstract}

\section{INTRODUCTION}

Piezoresponse atomic force microscopy is used to study the inverse piezoelectric effect on thin films non-destructively with a very high lateral resolution.

For this purpose, an AC electric field is applied to the specimen through a conductive SPM tip. The specimen's vertical and lateral response is measured with the microscope's optical detector (see figure 1). The response is then analyzed for the amplitude and the phase shift using a lock-in amplifier. By operating the SPM in contact mode [6], a surface map of the piezoelectric response amplitudes and -phases is created.

When using a regular cantilever far below its contact resonance frequency, the measured amplitude of the cantilever corresponds directly to the amplitude of the specimen.

The response amplitudes of the specimen are often in the order of magnitude of $10^{-12} \mathrm{~m}$. In order to overcome the relatively low signal to noise ratios (about 3-5), either higher AC electric fields have to be applied or an actuation frequency close to the contact resonance frequency of the cantilever has to be used. At

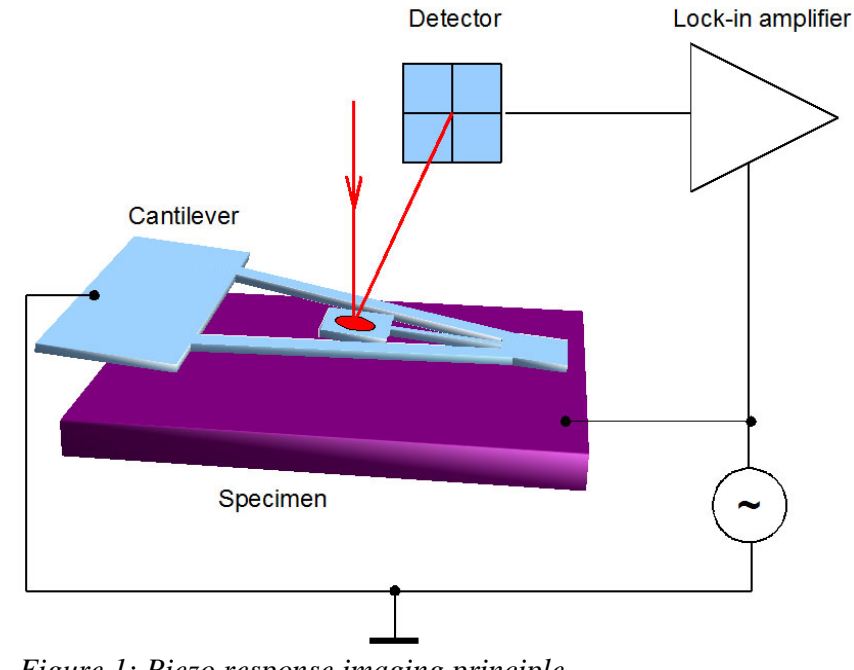

Figure 1: Piezo response imaging principle contact resonance one benefits from the higher transfer function of the cantilever in bending. Both measures directly influence the output amplitude and the signal-to-noise ratio of the measurement [7]. However, number of times the required AC electric fields for a good signal-to-noise however are in the same order of magnitude to create polarization in the specimen, thereby affecting the measurement.

The signal levels can be highly improved by operating the cantilever close to its contact resonance frequency [8]. The interpretation of the results obtained with this method though is quite complex. Since in this mode in addition to the piezoelectric response, the mechanical response of the cantilever couples into the measured amplitude and phase signals.

\section{CANTILEVER DESIGN}

One measure to enhance the signal-to-noise ratio is the use of a different cantilever architecture that optimizes the mechanical

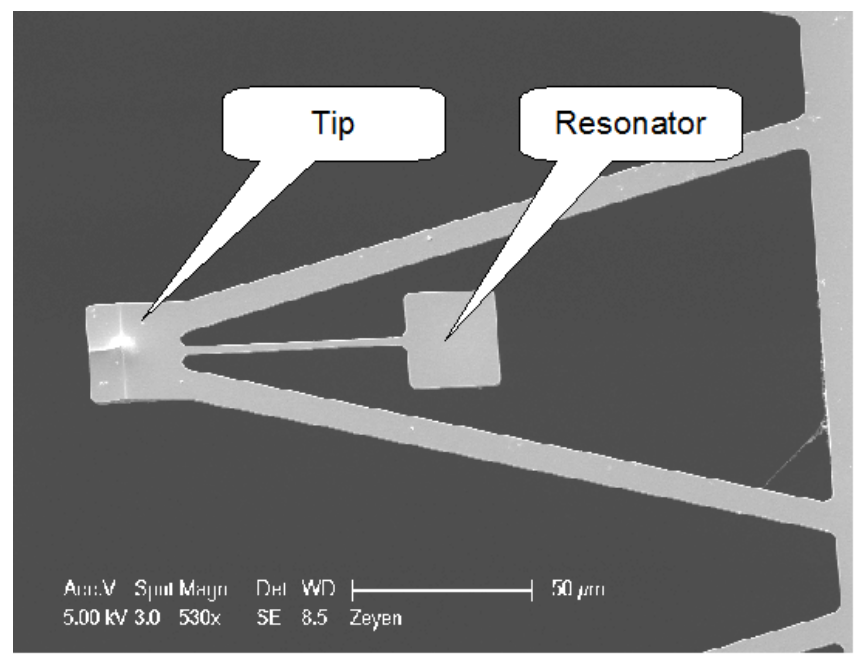

Figure 2. Preamplifying piezo response cantilever transfer function.

Figure 2 summarizes the basic design of the cantilever. It consists of a common triangular cantilever with its tip at the front end, and a resonator, attached via a coupling spring. This resonator, along with the coupling spring, serves as a tuning fork that reacts to (usually sample induced) movements in the tip in both vertical and lateral direction (compare [10]).

The laser of the atomic force microscope is focused onto the resonator during the measurements. Since the topographical features of the sample are scanned at a much lower rate than the resonator's frequency (in the order of $1 \mathrm{kHz}$ ), the angular deflection changes of the tip due to the topography get directly transferred to the resonator and can be read out and used for the SPM's feedback control signal.

Since the frequency of the applied AC signal corresponds to the resonator's resonance frequency, the periodic mechanical response of the specimen gets maximally amplified by this 
cantilever. It should be noted, that the measurements need a number of periods to settle as for any harmonic oscillator.

\section{FABRICATION}

The cantilever was fabricated using standard processes using a Silicon on Insulator (SOI) wafer. The process is summarized in figure 3. In the first step, the tip geometry was transferred onto a hard mask of SiO2, then isotropically etched in a SF6- plasma and sharpened using low temperature thermal oxidation at $900^{\circ} \mathrm{C}$. The resulting oxide was used as a hard mask for the overall cantilever contour shape, which was subsequently etched into the silicon with an anisotropic deep reactive ion etch step using $\mathrm{CHF}_{3}$. After patterning the backside consisting of $\mathrm{SiO}_{2}$ and $\mathrm{SiN}_{\mathrm{x}}$ with $\mathrm{CHF}_{3}$ and $\mathrm{CF}_{4}$ and passifying the front-side with a rubber-based coating (Brewer Science Protek B3 [11]) the backside was anisotropically wet etched with $\mathrm{KOH}$ until the oxide etch stop layer was hit. In a final step the coating was solved with acetone. The etch stop layers as well as the hard masks were removed with buffered HF; the wafer cleaned with an oxide plasma and coated on the front- and back side with a thin layer of titanium and gold - the front side for electric conductivity and the backside for better optical properties.

These cantilevers can be operated in a regular SPM with the laser focused on the resonator.

\section{EXPERIMENTS ON PIEZOELECTRIC MATERIALS}
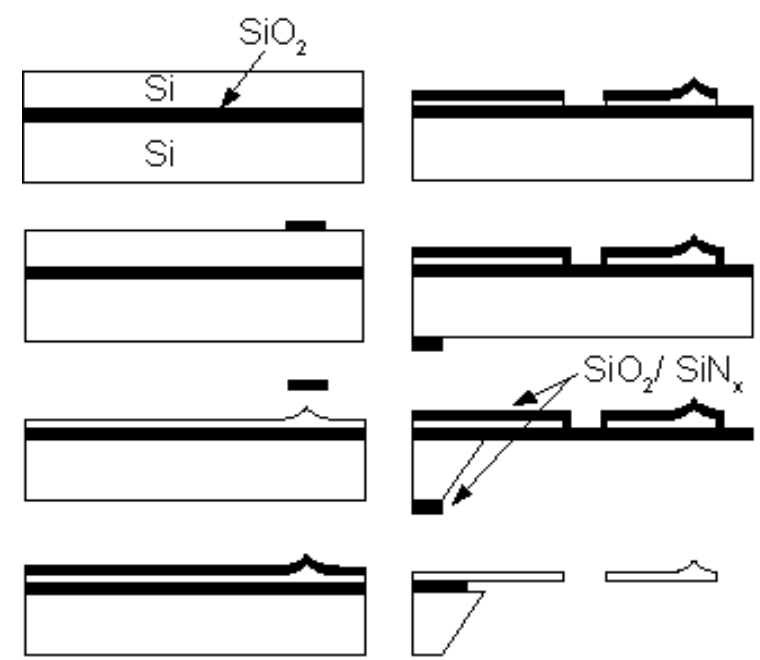

Figure 3. Fabrication process

The cantilever was tested with a Veeco Dimension V microscope using a Nanoscope 5 controller, sampling a periodically poled Lithium Niobate (PPLN) material. By applying an AC voltage between the tip and the sample, the mechanical reaction of the sample was characterized.

Figure 4 shows the vertical (ver.) and torsional (hor.) dynamic behavior of the cantilever while in contact with the specimen for the case where the laser was focused on the tip (tip) and then on the resonator (res.). The measurements indicate a total mechanical preamplification in the vertical direction of over 100 . This was obtained by operating at the resonator's resonance.

The ratio of 100 was obtained by dividing vertical resonator deflection amplitude at resonance with the tip deflection amplitude out of resonance.

This curve was used to quantify the mechanical response: In the first step, the laser was focused onto the tip to statically measure the overall optical lever sensitivity (with a force- deflection curve on a hard sample) and also its piezo response amplitude far below the cantilever's contact resonance frequency.

In the second step the laser was focused onto the resonator to measure the resonator optical lever sensitivity (again, with a force

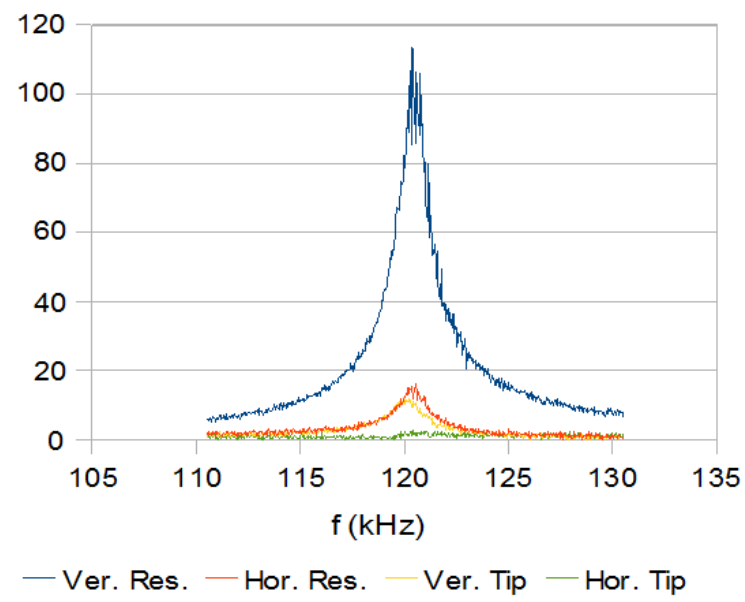

Figure 4. Dynamic behavior of the cantilever

curve on a hard sample) and the piezo response amplitude at the resonator's resonant frequency. Since the true piezo response amplitude is known from the first step of the calibration, the amplitude obtained with the laser on the resonator was used to determine the piezoresponse sensitivity.

Special caution had to be applied to the interpretation of the quantified signals however, as the response signal does not only contain the mechanical response of the piezoelectric material, but also an electrostatic contribution from the applied AC bias [8] and geometric effects of a locally induced piezoelectric strain [9]. The interpretation is still under investigation and will be a part of a future publication.

Figure 5 illustrates the vertical amplitude response of the cantilever close to (a) and far off resonance (b) using the same actuation amplitude to create the electric AC field in the sample. In (a), the domains and the domain changes between oppositely poled areas of the PPLN sample can be clearly seen as a small drop in the amplitude, whereas in (b) the overall amplitude ranges close to the noise level. The amplitude ranges used to create the images reflect the overall harmonic amplification of the cantilever.

a)

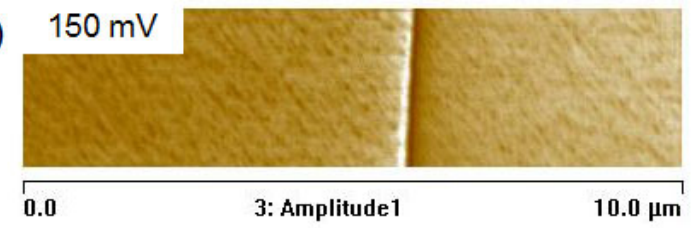

b)
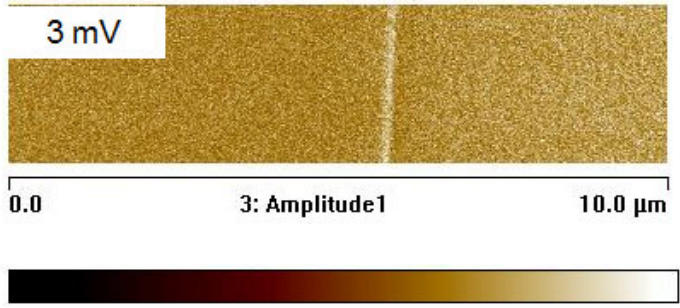

Figure 5. Amplitude response behavior for resonant and out-ofresonant actuation 
a)

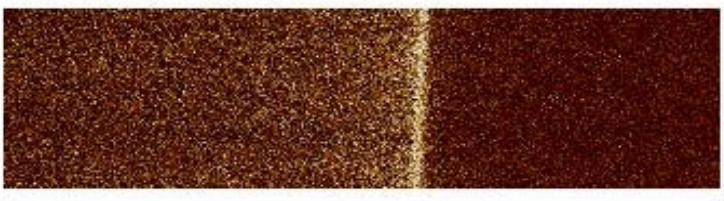
0.0

4: Phase1

$10.0 \mu \mathrm{m}$

b)

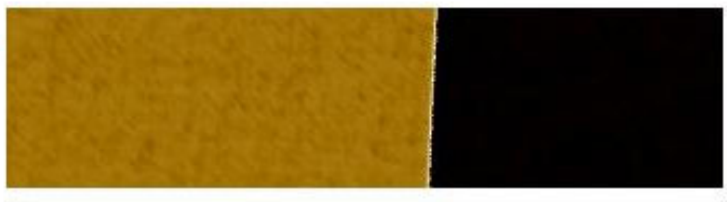

0.0

4: Phase1

$10.0 \mu \mathrm{m}$

c)

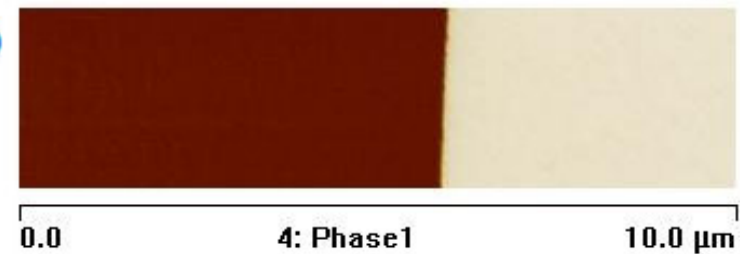

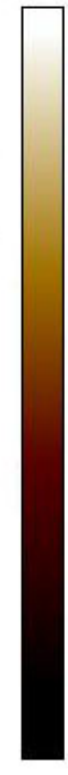

Figure 6. Phase response of the cantilever for a) out-of-resonance, b) subresonant and c) superresonant actuation

Figure 6 summarizes the phase images for an actuation of the sample out of resonance (a), slightly smaller than resonance (b) and slightly above resonance (c), using the same actuation voltage for all three experiments.

By comparing the phase contrast of the histograms close to resonance ( (b) and (c) ) to the histogram using an actuation out of resonance (a), the improvement of the signal-to-noise ratio can be demonstrated. For imaging phase maps of specimen close to the cantilever's contact resonance frequency it is important to take into consideration the phase shift in the response introduced by the cantilever. (b) and (c) show the phase shift when imaging the same part of the sample close below and above resonance. To obtain absolute quantitative results for phase image studies, the phase shift of the overall system, including the cantilever, has to be considered.

During our tests, the tip sample AC voltage at resonance could be reduced one order of magnitude (compared to previous cantilevers), overall to $200 \mathrm{mV}$ while still achieving a good signalto-noise ratio. Figure 7 shows the resulting picture with a signal amplitude of $7.5 \mathrm{mV}$. Applying the quantitative calibration scheme described above, we conclude that the specimen response amplitude in this figure was less than 5pm. For comparison previously used commercial SCM-PIT cantilevers [12] exhibited a signal amplitude of $12 \mathrm{mV}$ for a $10 \mathrm{~V}$ applied AC bias that corresponded to approximately $40 \mathrm{pm}$ sample response (the $5 \mathrm{pm}$ response amplitude is an upper bound. The discrepancies between the result and the comparison can originate from the elevated noise level when directly measuring response amplitudes far out of resonance as well as different conductivities, tip shapes of the cantilevers under study).

\section{SUMMARY}

We have designed, fabricated and tested a new cantilever for piezo response imaging in contact mode by optimizing the cantilever geometry for a maximized transfer function. We have characterized the cantilever using periodically poled Lithium Niobate thin films and demonstrated an one order of magnitude increase in sensitivity and signal-to-noise ratio that can be either used to achieve higher response- or lower excitation amplitudes.
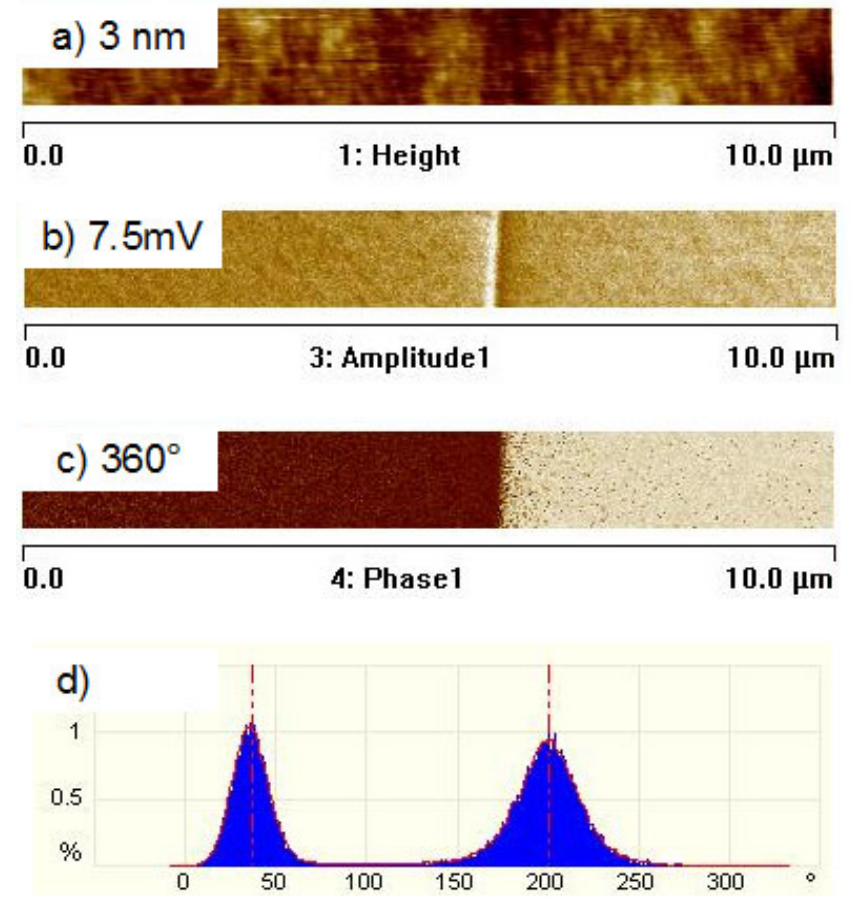

Figure 7. Results for specimen response amplitudes $<5 \mathrm{pm}$ 


\section{ACKNOWLEDGEMENTS}

This research was partially supported by a grant from the Center for Nanoscale Mechatronics \& Manufacturing, one of the 21st Century Frontier Research Programs, which are supported by Ministry of Science and Technology, KOREA

\section{REFERENCES}

[1] A. Gruverman, O. Auciello, H. Tokumoto: Imaging and control of domain structures in ferroelectric thin films via scanning force microscopy, Annu. Rev. Mater. Sci. No 28, 101-123 (1998)

[2] T. Tybell, C.H. Ahn, J.-M. Triscone: Control and Imaging of ferroelectric domains over large areas with nanometer resolution in atomically smooth epitaxial $\mathrm{Pb}\left(\mathrm{Zr}_{0.2} \mathrm{Ti}_{0.8}\right) \mathrm{O}_{3}$ thin films, Appl. Phys. Lett., Vol 72, No. 12, 23 (1998)

[3] C.S. Ganpule et al: Imaging three-dimensional polarization in epitaxial polydomain ferroelectric thin films, J. Appl. Phys., Vol 91, No. 3, 1 (2002)

[4] E. Soergel: Visualization of ferroelectric domains in bulk single crystals, Appl. Phys. B 81,729-752
[5] T. Jungk, A. Hoffmann, E. Soergel: Quantitative analysis of ferroelectric domain imaging with piezoresponse force microscopy, Appl. Phys. Lett. 89, 163507 (2006)

[6] G. Binne, C.F. Quate: Atomic Force Microscopy, Phys. Rev. Lett. Vol. 56, No. 9 (1986)

[7] K. Virwani, V. Kelley: Piezoresponse Atomic Force Microscopy Using a NanoScope V Controller, User Guide 013-444-000, Veeco Instruments

[8] C. Harnagea, A. Pignolet, M. Alexe, D. Hesse: Higher-Order Electromechanical Response of Thin Films by Contact Resonance Piezoresponse Force Microscopy, IEEE Transactions on Ultrasonics, Ferroelectrics and Frequency Control, Vol. 53, No. 12, 2309-2322 (2006)

[9] C. Harnagea, A. Pignolet, M. Alexe, D. Hesse: Piezoresponse scanning force microscopy: What quantitative information can we really get out of piezoresponse measurement on ferroelectric thin films? Integr. Ferroelectr. Vol 44, 113-124 (2002)

[10] B. Zeyen, KL Turner: Design and Test of a Novel Higher Harmonic Imaging AFM Probe With a Dedicated Second Cantilever for Harmonic Amplification, Transducers\& Eurosensors '07, 1545-1548 (2007)

[11] http://www.brewerscience.com/products/sm/productinformation/temporary-etch-protective-coatings/

[12] https://www.veecoprobes.com/probe_detail.asp?ClassID=103 